\title{
POPULATION I CEPHEIDS - THEORY AND OBSERVATION
}

\author{
R. S. STOBIE
}

Mount Stromlo and Siding Spring Observatory, Australian National University, Canberra, Australia

\begin{abstract}
The quantitative discrepancies between theory and observation with regard to population I Cepheids are discussed. The discrepancies cannot be reconciled by mass loss or by changes in the luminosity or effective temperature of the variables. It is concluded that the present pulsation models both linear and non-linear are in error.
\end{abstract}

\section{Introduction}

In this paper, I will concentrate on the disagreement between theory and observation with regard to population I Cepheids. Qualitatively, there is fair agreement between theory and observation in that evolutionary tracks predict the number-period distribution of observed Cepheids, unstable pulsation models are found in the observed strip and the light and velocity curves of non-linear pulsation models compare favorably with observation even in details such as bumps in the light curve. Quantitatively, however, a comparison of theory and observation reveals a number of discrepancies for which a variety of explanations have been proposed in the literature. Each discrepancy may be expressed in different ways and we choose here to express it in terms of the mass of a Cepheid.

The mass of a population I Cepheid may be calculated by at least three methods. (a) From the Cepheids in open clusters and associations; (b) from Cepheids with a modulated light curve and (c) from Cepheids with a bump in their velocity curve. Each method is discussed in turn and finally we comment on the apparent discrepancy in the transition period from fundamental to first overtone pulsation.

In what follows, $M$ and $R$ denote the mass and radius of a star in solar units.

\section{Calibration Cepheids}

Sandage and Tammann (1969) have listed 13 Cepheids which have been used to calibrate the zero point of the period-luminosity-color relation (PLC). These 13 Cepheids are considered to have the most accurately determined absolute magnitudes and unreddened colors. This enables the radius of each Cepheid to be calculated. Given $P$ and $R$, the theoretical PRM relation may be used to calculate the mass. The most complete derivation of the PRM relation for population I Cepheids is that given by Cox et al. (1972) but it is sufficient for our purpose to use the approximate relation for fundamental mode pulsators

$$
P_{0}=\alpha R^{7 / 4} M^{-3 / 4},
$$

where $\alpha$ is a constant ( $\alpha=0.022$, Christy, 1966). The mass derived in this way is called the pulsation mass, $M_{Q}$. 
The mass $M_{Q}$ is compared with the evolutionary mass, $M_{\mathrm{ev}}$, which is derived from the luminosity of evolutionary models during the core helium-burning stage. A systematic discrepancy is found for the 13 calibrating Cepheids and typically $M_{Q} / M_{\mathrm{ev}}=$ $=0.70$ (Cogan, 1970; Rodgers, 1970; Iben and Tuggle, 1972).

\section{Beat Cepheids}

The majority of population I Cepheids have a singly periodic light curve. However, there exists a subset of Cepheids of short period whose light curve is modulated. These Cepheids are indistinguishable from population I Cepheids with regard to their gravity and effective temperature (Rodgers and Gingold, 1973). The modulation may be interpreted as a superposition of two modes of radial pulsation which correspond to fundamental and first overtone (Stobie, 1970). Eight beat Cepheids have been analysed for their periodicities and they have similar properties. The period, $P_{0}$, lies in the range 2 to 4 days and the period ratio, $P_{1} / P_{0}$, lies in the narrow range 0.703 to 0.711 (Stobie and Hawarden, 1972). A knowledge of $P_{0}$ and $P_{1} / P_{0}$ is sufficient to calculate the mass of a beat Cepheid, $M_{\mathrm{b}}$.

Equation (1) may be rewritten

$$
Q_{0}=\alpha R^{1 / 4} M^{-1 / 4}
$$

where $Q_{0}=P_{0} R^{-3 / 2} M^{1 / 2}$. Elimination of $R$ from Equations (1) and (2) gives

$$
M_{\mathrm{b}}=\alpha^{6} P_{0} Q_{0}^{-7} \text {. }
$$

The value of $Q_{0}$ may be derived from the ratio $P_{1} / P_{0}$ and is almost model independent (Fitch, 1970). The observed ratio of $P_{1} / P_{0}=0.707 \pm 0.004$ leads to $Q_{0}=0.042$ \pm 0.001 from Equation (3) of Fitch (1970). With $\alpha=0.022, Q_{0}=0.042$ and $P_{0}=3$ days the mass of a typical beat Cepheid is $M_{b}=1.5$. This is a factor 3 lower than the expected evolutionary mass.

Similar results have been obtained (Petersen, 1973) by considering the position of the beat Cepheids in the $P_{0}-P_{1} / P_{0}$ diagram. Rodgers (1970) and Schmidt (1972) also pointed out this discrepancy although they expressed it in a different form.

\section{Bump Cepheids}

Another method of determining the mass is to consider Cepheids with an observed bump in their velocity curve. Similar bumps have been found in the non-linear pulsation models (Christy, 1970). If the bump is defined as the second (not secondary) feature in the velocity curve, then the phase $\varphi$ of the bump satisfies

$$
P_{0} \varphi=\beta R,
$$

where $\beta$ is a constant $(\beta=0.25$, Fricke et al., 1972).

Eliminating $R$ from Equations (1) and (4) we derive the bump Cepheid mass

$$
M_{\varphi}=\alpha^{4 / 3} \beta^{-7 / 3} P_{0} \varphi^{7 / 3} \text {. }
$$


With $\alpha=0.022, \beta=0.25$ and observed values of $P_{0}$ and $\varphi$, we find $M_{\varphi} / M_{\mathrm{ev}}=0.6$ (Fricke et al., 1972).

\section{Transition Period}

The results of non-linear pulsation models of population I Cepheids (Stobie, 1969) have indicated that the transition period between fundamental and first overtone pulsation occurs at $P_{\mathrm{tr}} \approx 7$ days. This conflicts with the observed transition period of about 3 days, which is identified with the region of the beat Cepheids and with the transition between the low amplitude sinusoidal light curves and the asymmetric high amplitude light curves (Gaposchkin and Gaposchkin, 1966). It is not clear how serious this discrepancy is in view of the suggested finite width of the transition region (van Albada and Baker, 1973) and the relationship between the linear and non-linear transition regions (Cox et al., 1972). Further work, particularly with the non-linear pulsation models, is required to clarify the theoretical transition region and to understand what parameters affect it.

\section{Discussion of Discrepancies}

The discrepancy between $M_{Q}$ and $M_{\mathrm{ev}}$ (Section 2) is the one most frequently discussed in the literature. A number of reasons have been advanced as to the cause of this discrepancy, e.g. mass loss, Hyades distance modulus, $T_{\mathrm{e}}-(B-V)$ transformation and errors in pulsation models (see for example, Cogan, 1970; Rodgers, 1970; Fricke et al., 1972; Iben and Tuggle, 1972; Schmidt, 1972). We hope to show by consideration of the other discrepancies (Sections 3,4 and 5) that errors in the pulsation models is the most likely explanation.

The discrepancies $\left(M_{Q}, M_{\mathrm{ev}}\right)$ and $\left(M_{\mathrm{b}}, M_{\mathrm{ev}}\right)$ may be derived from the results of either linear or non-linear pulsation theory. However, the discrepancies $\left(M_{\varphi}, M_{\mathrm{ev}}\right)$ and $\left(P_{\mathrm{tr}}\right)$ depend entirely on the results of non-linear pulsation theory. The calculation of $M_{Q}$ is dependent on knowing the absolute magnitude and unreddened color of a Cepheid and the relations transforming these quantities into luminosity and effective temperature. To this extent, the results in Section 2 could possibly be explained by errors in the distance modulus, reddening and transformation relations. However, discrepancies $\left(M_{\mathrm{b}}, M_{\mathrm{ev}}\right),\left(M_{\varphi}, M_{\mathrm{ev}}\right)$ and $\left(P_{\mathrm{tr}}\right)$ are essentially independent of these errors, i.e. altering $L$ and $T_{\mathrm{e}}$ will not remove the discrepancy. Furthermore the three masses $M_{Q}$, $M_{\varphi}$ and $M_{\mathrm{ev}}$ cannot all be made to agree by any change in $L$ or $T_{\mathrm{e}}$ (Fricke et.al., 1972).

Thus the discrepancies considered together indicate that something is wrong with present pulsation models both linear and non-linear. The discrepancy $\left(M_{\mathrm{b}}, M_{\mathrm{ev}}\right)$ shows this most clearly as the principal uncertainty in Equation (3) is $\alpha$. As this mass determination is most sensitive to the constants in the theoretical relations, it is not surprising that the largest discrepancy should occur here if the pulsation models are in error.

The results also show that mass loss by itself cannot explain all of the discrepancies because the masses derived from the pulsation model results $\left(M_{Q}, M_{\mathrm{b}}\right.$ and $\left.M_{\varphi}\right)$ are 
themselves inconsistent. Hence although mass loss cannot be excluded, it is premature to invoke it as an explanation of any of the discrepancies until consistency is achieved.

\section{Errors in Pulsation Models}

By errors in pulsation models we do not mean that pulsation theory is incorrect. Indeed pulsation theory can explain so many properties of Cepheid variables that it is considered basically correct. Rather the error may lie in the input physics (e.g. opacity table) which will affect the model structure of the Cepheid.

A preliminary investigation of arbitrary changes in the opacity table (Stobie, unpublished) has revealed that it is possible to find a single opacity change which can account for all three mass discrepancies. The opacity change required is uncomfortably large being up to a factor 4 greater than the Cox and Stewart (1965) opacities. However, relative to the opacities of Carson et al. (1968) this factor would not appear so large as their opacities at some densities and temperatures are up to a factor 3 greater than those of Cox and Stewart (1965). It would be most interesting to recalculate the properties of pulsation models if and when a complete opacity table is available using the results of Carson et al. (1968).

\section{References}

Albada, T. S. van and Baker, N.: 1973, Astrophys. J. 185, 477.

Carson, T. R., Meyer, D. F., and Stibbs, D. W. N.: 1968, Monthly Notices Roy. Astron. Soc. $140,483$.

Christy, R. F.: 1966, Ann. Rev. Astron. Astrophys. 4, 353.

Christy, R. F.: 1970, J. Roy. Astron. Soc. Can. 64, 8.

Cogan, B. C.: 1970, Astrophys. J. 162, 139.

Cox, A. N. and Stewart, J. N. : 1965, Astrophys. J. Suppl. 11, 22.

Cox, J. P., Castor, J. I., and King, D. S. : 1972, Astrophys. J. 172, 423.

Cox, J. P., King, D. S., and Stellingwerf, R. F.: 1972, Astrophys. J. 171, 93.

Fitch, W. S.: 1970, Astrophys. J. 161, 669.

Fricke, K., Stobie, R. S., and Strittmatter, P. A.: 1972, Astrophys. J. 171, 593.

Gaposchkin, C. P. and Gaposchkin, S.: 1966, Smithsonian Contrib. Astrophys. 9, 1.

Iben, I. and Tuggle, R. S. : 1972, Astrophys. J. 173, 135.

Petersen, J. O.: 1973, Astron. Astrophys. 27, 89.

Rodgers, A. W.: 1970, Monthly Notices Roy. Astron. Soc. 151, 133.

Rodgers, A. W. and Gingold, R. A.: 1973, Monthly Notices Roy. Astron. Soc. 161, 23.

Sandage, A. and Tammann, G. A.: 1969, Astrophys. J. 157, 683.

Schmidt, E. G.: 1972, Astrophys. J. 176, 165.

Stobie, R. S. : 1969, Monthly Notices Roy. Astron. Soc. 144, 511.

Stobie, R. S. : 1970, Observatory 90, 20.

Stobie, R. S. and Hawarden, T.: 1972, Monthly Notices Roy. Astron. Soc. 157, 157.

\section{DISCUSSION}

Cox: I was absolutely amazed recently to see a paper by Böhm-Vitense where she tried to get the masses and helium content of some stars in globular clusters and she used Christy's blue edges and Iben's blue edges. The difference only is that Christy has a formula for the opacity and Iben has got a detailed table. By using these two sets of blue edges, she got the helium content in these globular clusters to vary a lot. I always thought that Christy had made a pretty good fit. My question is: have you obtained your $\delta \log \kappa$ from Christy's fit or from a very detailed table such as Iben has produced? 
Stobie: It was relative to a coarse table which you produced, coarse in the sense that it is a coarse grid.

Cox: My point is - you are right. There seems to be sensitivity and I was amazed at it.

Stobie: We did originally consider just absolute constant changes, say just multiplying $\kappa$ by a factor 2 , and that makes virtually no change to the periods that you get for a given star of given mass and radius, You have got to make a change in the gradient before you got some substantial effects. The opacity change required to give agreement is up to a factor 5 different from the original opacity table and this is getting a bit drastic.

Rodgers: In changing the gradient of the opacity, have you done any non-linear calculations to see whether it changed the transition periods in the right direction?

Stobie: All these changes I considered, made absolutely no difference at all to the transition periods and that is one reason why I have been reluctant to publish anything, because I could not get anything to change that transition period and it always was coming out near seven days. The discrepancy for that must be some other cause which I do not understand.

Rodgers: Could I make one comment; saying that Dr Cox throughout this discussion has treated mass and helium content as two variables which you could play with and compensate. I would like to make a point, as a simple minded spectroscopist. To change helium abundances, in what Professor Eggen would call medium period young disc population variables, is no small matter to people who have made lots and lots of detailed analyses of helium abundances in B stars. I do not feel that the Kiel group, for instance, would view with similar cheerfulness, the idea that you can throw helium abundance around in the kind of way Dr Cox might like.

Buscombe: Isn't it different in the interior than it is on the skin?

Cox: I am not talking about very much. I am talking about the difference between 0.35 and 0.45 . There is a recent Russian paper where he took Stobie's blue edges, and he matched and he got $45-50 \%$ helium in these stars. The same thing could be done with normal evolutionary masses which I have talked about this afternoon.

Stobie: I would place little reliance on the blue edges because there are problems with the boundary conditions. The boundary conditions were obviously so sensitive you have got to be sure that you do it consistently. The difference was just so much you could not say what the helium abundance was with any certainty.

Consider the case where there is an opacity change which increases with temperature - this may seem pretty arbitrary, but it would perhaps not be inconsistent with the opacity changes which Stibbs, Carson and Hollingsworth have been considering. Admittedly they have done no opacities computation in the actual temperature and density region here but they definitely found factors of 2 or even 3 , at about $10^{5}$ and $10^{6} \mathrm{deg} \mathrm{K}-$ higher than Cox and Stewart opacities. If you believe that the opacities at lower temperatures are contributed mostly by hydrogen and helium, which we think we know well, you would expect then that there must be some gradient somewhere along here if you believe their results. Do you have any comment on that?

Cox: I guess the answer to that is that I don't believe every syllable. The opacities are very uncertain, there is no doubt about that. 